\title{
Economic Policy Uncertainty and Local Carbon Emission Trading: A Multifractal Analysis from US and Guangdong
}

\author{
Ruwei Zhao \\ School of Business, Jiangnan University, Wuxi, Jiangsu 214122, China \\ Correspondence should be addressed to Ruwei Zhao; zrwjnu@jiangnan.edu.cn
}

Received 12 May 2021; Accepted 23 June 2021; Published 5 July 2021

Academic Editor: Lei Xie

Copyright (C) 2021 Ruwei Zhao. This is an open access article distributed under the Creative Commons Attribution License, which permits unrestricted use, distribution, and reproduction in any medium, provided the original work is properly cited.

\begin{abstract}
This paper investigates the long-term dynamic cross-correlation evolution between US economic policy uncertainty index (USEPU) and Guangdong carbon emission trading price (GDCP) from the multifractal detrended cross-correlation analysis (MFDCCA) perspective. With the calculation of correlation statistics and fluctuation function, the beginning procedures of MFDCCA, we find that the cross-correlation between USEPU and GDCP is significant and presents power law property. Also, with the Hurst exponent, we find that the long-horizon correlations between series are persistent. Moreover, we perform Rényi exponent and spectrum singularity check. The empirical findings reveal that the all the correlations are of multifractality and the correlation of GDCP holds the highest degree.
\end{abstract}

\section{Introduction}

It is well acknowledged that climate change is the most challenging task the human being has ever encountered. As an effective solution for carbon emission reduction, carbon emission trading market has been widely accepted by many countries [1]. With this view, numerous studies contribute to the carbon emission trading field with regard to the mechanism and consequences of carbon emission trading market [2-14]. Also, as an ideal proxy for the measurement of macroeconomic fluctuation, economic policy uncertainty index has been commonly employed by the financial researchers [15-30]. Due to the huge impact of economic policy changes, several studies link economic policy uncertainty with carbon emissions to check the correlations between them. Adams et al. [31] took an investigation of economic policy uncertainty and carbon emission along with energy consumption through the autoregressive model. They find that compared with energy consumption, the influence of economic policy uncertainty to carbon emission can be ignored. Yu et al. [32] carried out a study regarding the economic policy uncertainty (EPU) and firm carbon emissions from provincial levels. They found that with rise in the economic policy uncertainty, firms intend to employ cheap and low quality fossil fuels, which would result in an increase in carbon emission. However, these studies usually depict linear property between variables and take little concern over carbon emission trading market. Multiple research studies have recorded that it is hard for traditional statistical vehicle to discover the nonlinear correlation between series due to nonstationary evolution pattern of economic time series. [33-38]. With this view, researchers are trying to utilize multifractal analysis in modeling the nonlinear dynamic correlation between economic time series. Liu et al. [15] examined the cross-correlation between economic policy uncertainty and stock market volatility with multifractal analysis. They found that with the involvements of economic policy uncertainty and the multifractal model, the forecasting accuracy for future volatility can be greatly improved. Yao et al. [39] studied multifractal correlation among crude oil market, US stock market, and economic policy uncertainty. They found that the nonlinear long-range correlation between series is persistent and strong evidence for the existence of series correlation multifractality.

As the richest province of China, Guangdong weighs $10 \%$ of China total GDP and has been the economic champion among China's provinces over decades. Also, as a pioneer in China's reform and opening-up process and 
neighboring province of Hong Kong, Guangdong is the first province opening for foreign investment. With decades of development, the US has been the largest trade partner of China, indicating that considerable Guangdong firms engage in the Sino-US trade. Besides, as the first batch of pilot provinces in carbon emission trading, Guangdong is of the longest history and largest volume in carbon emission trading. With this view, we connect US economic policy uncertainty index, as the representative of US macroeconomic policy change, with the Guangdong carbon emission trading price to check whether US policy changes would demonstrate impact to local carbon emission trading. Also, as a common statistical vehicle for multifractality, we employ the MF-DCCA to check whether cross-correlation multifractality exists between US policy uncertainty and Guangdong carbon emission trading. With significant crosscorrelation statistics, we find the existence of long-horizon cross-correlation between US economic policy uncertainty and Guangdong carbon emission trading price. Additionally, we find that the cross-correlations between series are of power law property with the upward trend of fluctuation function with no regard to changes of scaling orders. Furthermore, we calculate the Hurst exponent to check the persistence of the correlation. We find that all the exponent values are above critical values, proving the persistence of correlation. Also, we find that none of the exponents are of constant values, confirming the cross-correlation multifractality. Moreover, we calculate the Rényi exponent and spectrum singularity as other checks for multifractality. We find that none of the Rényi exponent curves are of typical linear characters, confirming the multifractality existence. In addition, with the calculation of Hölder exponent difference, we find that Guangdong carbon price is of greatest difference, informing the richest multifractality.

Our study enriches the current literature from two dimensions. First, we utilize the novel US economic policy uncertainty index as an indicator for the US macroeconomic policy fluctuation and link it with Guangdong carbon emission trading price from the multifractal perspective. Particularly, we find that long-horizon cross-correlation between US economic policy uncertainty and Guangdong carbon emission trading price exists. Moreover, with the Hurst and Rényi exponent, we find that the cross-correlation between series demonstrates solid evidence for the multifractality existence. From this point of view, our empirical findings are in line with prior economic research studies related to multifractality analysis [39-45]. Second, our study originated from the multifractal perspective, enabling indepth investigation for the cross-correlation dynamics between economic policy uncertainty and carbon emission trading. Furthermore, as the most important economic zone in China, our findings would shed light on the carbon emission market risk management for not only Guangdong but also China policy makers and investors by avoiding the imported economic uncertainty.

The rest of this paper is organized as follows. Section 2 describes the data. Section 3 illustrates the multifractal crosscorrelation methodology. Section 4 shows the empirical results. Section 5 concludes the paper.

\section{Data}

Our data consisted of US economic policy uncertainty index and Guangdong carbon emission price. US economic policy uncertainty index is developed by Baker et al. [20]. We directly download the index from the websites (http:// policyuncertainty.com). As stated by the website, the US economic policy uncertainty index is constructed by counting article number of thousands of national and local newspapers archived in the Access World News' NewsBank service with three sets of terms. The first set refers to the terms economic or economy, such as monetary policy. The second set terms are uncertain or uncertainty, such as COVID-19. The third terms are related to government actions, such as deficit. The Guangdong carbon emission price is derived from the Wind Financial Services, a widely accepted financial data provider in China. For the US and Guangdong data pairing, we chose the sample time period from December 19, 2013, the earliest day of carbon emission trading, to April 29, 2021.

Table 1 presents the descriptive statistics of the US economic policy uncertainty index (USEPU) and Guangdong carbon emission trading price (GDCP). We can find from Table 1 that both GDCP and USEPU series present right-skew characters with greater averages of the GDCP and USEPU (22.57 and 120.82) over their medians (18.56 and 86.73). Also, the skewness of both series is positive (2.08 and 2.36), confirming the right-skew distribution findings. For the kurtosis, it is easy to find that the values of both series (7.89 and 9.45) are larger than 3, indicating sharp peak characteristics. Moreover, we utilize the prevailing Jarque-Bera tests to check whether both series demonstrate normality properties. We can find from Table 1 that JB statistics of both series (2555.47 and 3975.91) are much greater than zeros. As a result, the $p$ values of JB tests are zeros, presenting strong evidence of the non-normal distribution existence.

Figures 1 and 2 present the evolution patterns of the Guangdong carbon emission trading price and US economic policy uncertainty index. As shown in Figure 1, the GDCP reaches its peak at the beginning of the series. After that, the GDCP series experience continuous drop and bounce back at 2018. On the contrary, in Figure 2, the USEPU series demonstrate moderate fluctuation from 2013 to 2019. However, entering 2020, the USEPU raises sharply, which may be caused by the uncertainty of US election and heavier situation of COVID-19. Furthermore, both series patterns show strong rejections to normality distributions with apparent sharp peaks and fat tails, which are consistent with statistics presented in Table 1.

\section{Methodology}

In this section, we employ the prevailing multifractal cross-correlation analysis (MF-DCCA) as an instrument to investigate whether multifractality exists between the Guangdong carbon emission trading price (GDCP) and US economic policy uncertainty index (USEPU). First, we utilize the cross-correlation statistics proposed by 
TABle 1: Descriptive statistics.

\begin{tabular}{lcc}
\hline Variable & GDCP & USEPU \\
\hline Mean & 22.57 & 120.82 \\
Median & 18.56 & 86.73 \\
Maximum & 77.00 & 738.02 \\
Minimum & 8.10 & 10.92 \\
Std. Dev. & 11.97 & 103.60 \\
Skewness & 2.08 & 2.36 \\
Kurtosis & 7.89 & 9.45 \\
Jarque-Bera & 2555.47 & 3975.91 \\
Probability & $\leq 0.01$ & $\leq 0.01$ \\
$N$ & 1491 & 1491 \\
\hline
\end{tabular}

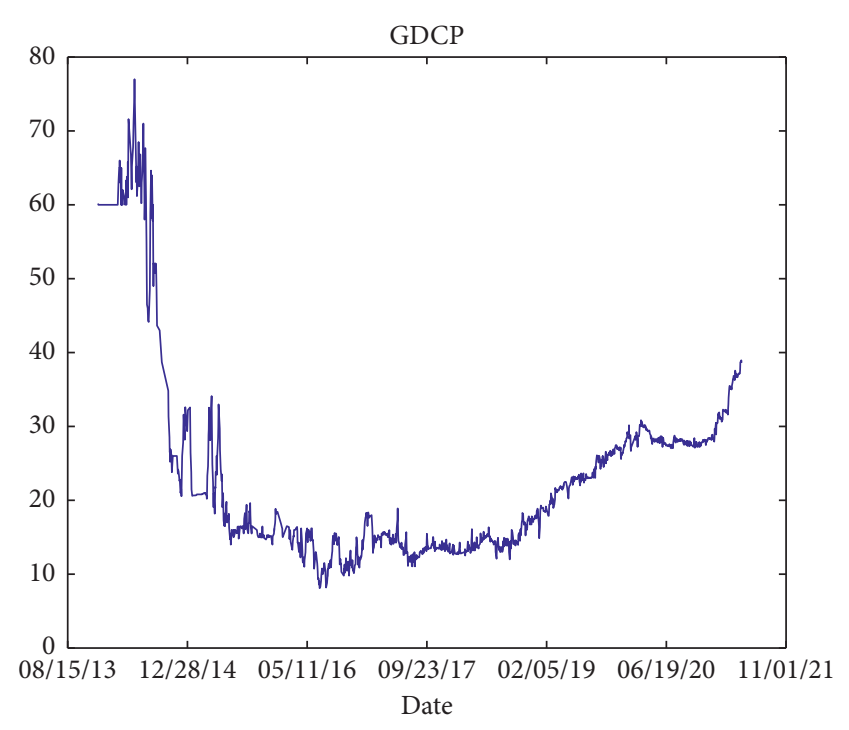

Figure 1: Evolution pattern of Guangdong carbon emission price (GDCP).

Podobnik and Stanley [46] to check the existence of the cross-correlation between the series in a qualitative way. Second, we apply the MF-DCCA approach to inspect if the correlations between series would vary conditionally on the changes of scaling orders, which would result in multifractality.

3.1. Cross-Correlation Analysis. To have a general view of cross-correlation between GDCP and USEPU, we first introduce a cross-correlation indicator proposed by Podobnik and Stanley [8]. The indicator $C_{i}$ is constructed through the following equation:

$$
C_{i}=\frac{\sum_{k=i+1}^{N} x_{k} y_{k-i}}{\sqrt{\sum_{k=1}^{N} x_{k}^{2} \sum_{k=1}^{N} y_{k}^{2}}}
$$

where $\left\{x_{k}\right\}$ and $\left\{y_{k}\right\}$ are responsible for two equal length time series.

With the involvement of cross-correlation indicator $C_{i}$, we further begin to construct the cross-correlation statistics. The detailed construction process is shown in the following equation:

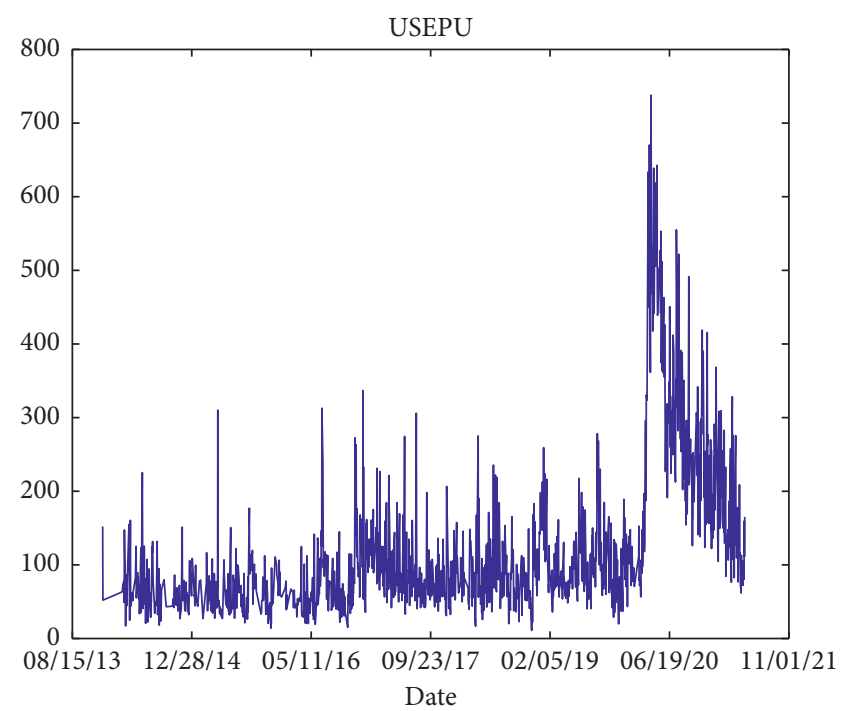

FIGURE 2: Evolution pattern of US economic policy uncertainty index (USEPU).

$$
Q_{\mathrm{cc}}(m)=N^{2} \sum_{i=1}^{m} \frac{C_{i}^{2}}{N-i},
$$

where $C_{i}$ refers to the cross-correlation indicator, $N$ is the total length of the series, and $m$ means the degree of freedom. We can indicate from the equation that the cross-correlation statistics $Q_{c c}(m)$ would follow the $\chi^{2}(m)$ distribution with $m$ degrees of freedom. To check if the cross-correlation between series is significant, we are required to compare the cross-correlation statistics $Q_{c c}(m)$ with chi-square critical value $\chi^{2}(m)$ under $m$ degrees of freedom proposed by Podobnik and Stanley [46]. If the value of the cross-correlation statistics $Q_{c c}(m)$ is above the critical value of chi-square $\chi^{2}(m)$, we believe a significant cross-correlation exists between the two time series.

3.2. MF-DCCA. Zhou [47] proposed the prevailing multifractal cross-correlation analysis (MF-DCCA) methodology to check the nonlinear cross-correlation between detrended time series. The MF-DCCA, derived from the detrended fluctuation analysis, is constructed through following five steps: 
Step 1. To eliminate the trending character of each series, we process the original time series $\left\{x_{k}\right\}$ and $\left\{y_{k}\right\}$, which are responsible for GDCP and USEPU, with a deduction of each series average, respectively. The detailed calculation process is presented by the following equation:

$$
\begin{aligned}
& X(i)=\sum_{k=1}^{i}\left(x_{k}-\bar{x}\right), \\
& Y(i)=\sum_{k=1}^{i}\left(y_{k}-\bar{y}\right),
\end{aligned}
$$

where $\bar{x}$ and $\bar{y}$ are the means of $\left\{x_{k}\right\}$ and $\left\{y_{k}\right\}, k=1,2$, $3, \ldots, N$. It is easy to deduce from equation (3) that $X(N)$ and $(N)$ are equal to zero.

Step 2. To have a microinvestigation of each series, we split the detrended profiles $X(i)$ and $Y(i)$ into $N_{s}$ nonoverlapping segments with the equal time lengths of $s$. The length interval $N_{s}$ is generated as follows:

$$
N_{s}=\operatorname{int} \frac{N}{s}
$$

where $N$ is the total length of observation and int stands for the integer function, collecting the maximum integer toward the real number. Nevertheless, in some occasions, the total length $N$ cannot be divided by scale $s$ with no reminder, which would leave a short part segment at the end of each profile. Considering this scenario, we regenerate the segment with the length interval $N_{s}$ from the last to the first value of the series for maximum usage of the whole time series. Thus, we can get two $N_{s}$ segments for each time series with repeated generation process.

Step 3. With divided $N_{s}$ segments, we start to investigate the local trend of each segment, $v$, through a polynomial fitness check. Therefore, the variance of each segment, denoted as $F^{2}(v, s)$, can be acquired through equations (5) and (6).

If $v=1,2,3, \ldots, N_{s}$, the variance of segment $v, F^{2}(\nu, s)$, is as follows:

$F^{2}(\nu, s)=\frac{1}{s} \sum_{i=1}^{s}\left|X((\nu-1) s+i)-p_{v}^{n}(i)\right| \bullet\left|Y((\nu-1) s+i)-p_{v}^{n}(i)\right|$.

If $v=N_{s}+1, N_{s}+2, N_{s}+3, \ldots, 2 N_{s}$, the variance of segment $v, F^{2}(\nu, s)$, is as follows:

$$
F^{2}(\nu, s)=\frac{1}{s} \sum_{i=1}^{s}\left|X\left(N-\left(\nu-N_{s}\right) s+i\right)-p_{v}^{n}(i)\right| \bullet\left|Y\left(N-\left(\nu-N_{s}\right) s+i\right)-p_{v}^{n}(i)\right|
$$

where $p_{v}^{n}(i)$ is the $n$-th order polynomial fitness check of segment $v$.

Step 4. To have a measurement of nonlinear correlation between series, we calculate the $q$-th order of the fluctuation function by averaging variances of all the detrended segments. The construction processes of the fluctuation function are presented by equations (7) and (8).

If $q \neq 0$,

$$
F_{q}(s)=\left[\frac{1}{2 N_{s}} \sum_{v=1}^{2 N_{s}}\left[F^{2}(v, s)\right]^{q / 2}\right]^{1 / q}
$$

If $q=0$,

$$
F_{0}(s)=\exp \left[\frac{1}{4 N_{s}} \sum_{v=1}^{2 N_{s}} \ln \left[F^{2}(\nu, s)\right]\right]
$$

It is easy to learn from equations (7) and (8) that the moving trend of fluctuation function $F_{q}(s)$ lies on the selection of the time length $s$ under given value of $q$. In addition, to have a deep understanding of multifractal property between series, we repeat procedures from No. 2 to No. 4 upon various selections of $s$ and finally draw forth our last fifth procedure.

Step 5. With the multiselection of time length s, fluctuation function $F_{q}(s)$ moving trend could be observed by the gradient of the $\log -\log$ plot of $F_{q}(s)$ versus $s$ conditional on various selections of scaling order q. If a long-term multifractal cross-correlation character could be detected between the detrended series $X(i)$ and $Y(i)$, the fluctuation function $F_{q}(s)$ would demonstrate a power law relationship as presented in the following equation:

$$
F_{q}(s) \sim s^{H_{X Y}(q)}
$$


where $H_{X Y}(q)$ is on behalf of the gradient of the log-log plots of $F_{q}(s)$ conditional on the variation of scaling order $q$ and $H_{X Y}(q)$ is estimated by the ordinary least square regression.

We can learn from equation (9) that the gradient of fluctuation function, $H_{X Y}(q)$, varies upon change of scaling order q. Also, when $q=2$, if the generalized scaling exponent $H_{X Y}(2)$ is larger than 0.5 , a long-term and consistent cross-correlation between detrended series $X(i)$ and $Y(i)$ would be confirmed. Nevertheless, if the scaling exponent of $H_{X Y}(2)$ is smaller than 0.5 , we believe the cross-correlation between detrended series $X$ (i) and $Y(i)$ is of no consistence. Also, if the scaling exponent $H_{X Y}$ (2) equals 0.5 , no significance of the cross-correlation between detrended series $X(i)$ and $Y(i)$ would be founded. With the consideration of the broad application, $H_{X Y}(2)$ is widely recognized as the generalized Hurst exponent. With this view, we define that if the scaling exponent $H_{X Y}(q)$ equals to a constant with no regard to any change of scaling order $q$, the cross-correlation between series is monofractal. Otherwise, we regard the cross-correlation between detrended series as multifractal. Moreover, we could learn from equations (3) and (5) that when $q$ is larger than zero, the segment $v$ works as a core factor in valuing fluctuation function $F_{q}(s)$ with the large variance of $F^{2}(v, s)$. With this view, when $q$ is over zero, the exponent $H_{X Y}(q)$ would be responsible for the large fluctuation scaling property. Conversely, when $q$ is below zero, the exponent $H_{X Y}(q)$ would be the indicator for the small fluctuation scaling property.

With the Hurst exponent $H_{X Y}(q)$, we start to construct the Rényi exponent, the key proxy for the multifractality investigation between series. The exponent is denoted as $\tau_{X Y}(q)$. The calculation process is as follows:

$$
\tau_{X Y}(q)=q H_{X Y}(q)-1 .
$$

Moreover, to enhance the credibility of multifractality, we perform the singularity spectrum, marked by $f_{X Y}(\alpha)$, as an additional check. With the Legendre transformation, the singularity spectrum, $f_{X Y}(\alpha)$, can be obtained by the following equations, with the participation of the Rényi exponent, $\tau_{X Y}(q)$, and the Hölder exponent $\alpha_{X Y}$. The detailed calculation process of $f_{X Y}(\alpha)$ is as follows:

$$
\begin{aligned}
\alpha_{X Y} & =\tau_{X Y}^{\prime}(q), \\
f_{X Y}(\alpha) & =q \alpha_{X Y}-\tau_{X Y}(q),
\end{aligned}
$$

where $\alpha_{X Y}$ is the Hölder exponent. As the key parameter in the singularity spectrum analysis, $\alpha_{X Y}$ is the representative of the strength of the singularity spectrum. With this view, we employ the strength difference or the spectrum width, $\Delta \alpha_{X Y}$, as the measurement for multifractality degree, where $\Delta \alpha_{X Y}=\alpha_{X Y \max }-\alpha_{X Y \text { min }}$. We can infer from the equation that a larger value of $\Delta \alpha_{X Y}$ would indicate a larger degree of multifractality.

\section{Empirical Results}

4.1. Cross-Correlation Test. In this section, to have a basic knowledge of statistical correlation between Guangdong carbon emission trading price and US economic policy uncertainty, we employ equations (1) and (2) to calculate the cross-correlation statistics, $Q_{c c}(m)$, as an instrument to investigate the correlation significance between series. Figure 3 shows the cross-correlation statistics, $Q_{c c}(m)$, and its corresponding chi-square critical value $\chi^{2}(m)$ at $5 \%$ significant level is depicted by green and red lines conditional on the degrees of freedom varying from 1 to $N-1$. We can find from Figure 3 that the cross-correlation statistic $Q_{c c}(m)$ demonstrates clear edge over its corresponding chi-square critical value $\chi^{2}(m)$ by noticeable distances between variables, which would strongly reject the null hypothesis and prove the long-term cross-correlation existence between GDCP and USEPU.

4.2. Multifractal Detrended Cross-Correlation Analysis. With significant correlation statistics, $Q_{\mathrm{cc}}(m)$, as presented in Figure 3, we could infer a long-term correlation between Guangdong carbon emission trading price and US economic policy uncertainty in a qualitative way. However, to have a detailed quantitative investigation of the nonlinear correlation character between Guangdong carbon emission trading price and US economic policy uncertainty, we utilize the multifractal detrended cross-correlation analysis proposed by Zhou [47] as the instrument. To perform the analysis, we first follow the work of Zhang et al. [48] by setting scaling order $q$ from -10 to 10 with an interval of 1 . Also, we can infer from the illustration in Section 3.2 that if scaling order $q$ is smaller than zero, we believe two time series demonstrate weak fluctuation. On the contrary, the time series show strong fluctuation. Figure 4 plots the log-log fluctuation function $F_{q}(s)$ evolution pattern conditional on the time length of $s$ with scaling orders from -10 to 10 between Guangdong carbon emission trading price and US economic policy uncertainty (GDCP and USEPU). It is easy to find that all of the lines demonstrate similar evolution patterns by moving upward upon gradual increase in time length $s$ with no regard to the changes of scaling orders, indicating a long-term power law correlation existence between GDCP and USEPU.

Also, to have an investigation of cross-correlation persistence and multifractality between Guangdong carbon emission trading price and US economic policy uncertainty series, we further calculate the Hurst exponent with the variation of scaling order $q$ as presented in Figure 5. In Figure 5, we can find that the Hurst exponent values of the Guangdong carbon emission trading price and US economic policy uncertainty (GDCP-USEPU cross-correlation), the Guangdong carbon emission trading price (GDCP), and the US economic policy uncertainty (USEPU) are all above the critical values (0.5), providing strong evidence that the crosscorrelation of GDCP-USEPU, the correlation within GDCP series, and the correlation within USEPU series are persistent. In addition, we can find from Figure 5 that GDCPUSEPU and GDCP present downward trend with decreased Hurst exponent values upon the rise of scaling order $q$. However, for USEPU, the line goes down with gradual increase in scaling order $q$ from -10 to -2 . After that, the line 


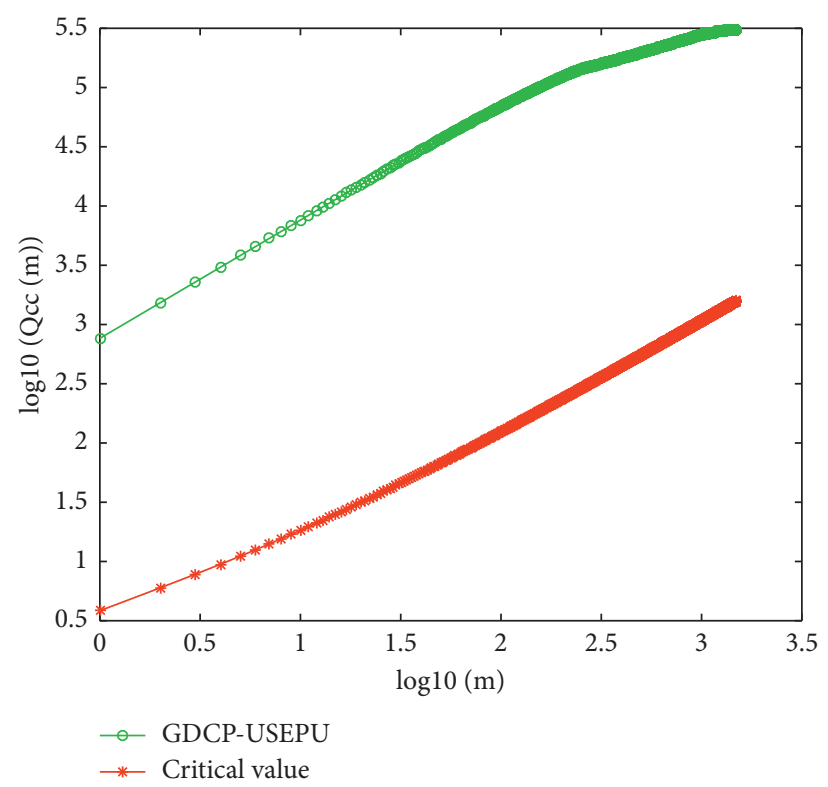

FIGURE 3: $\log -\log$ plot of the cross-correlation statistics $\left(Q_{c c}(m)\right)$ for GDCP and USEPU.

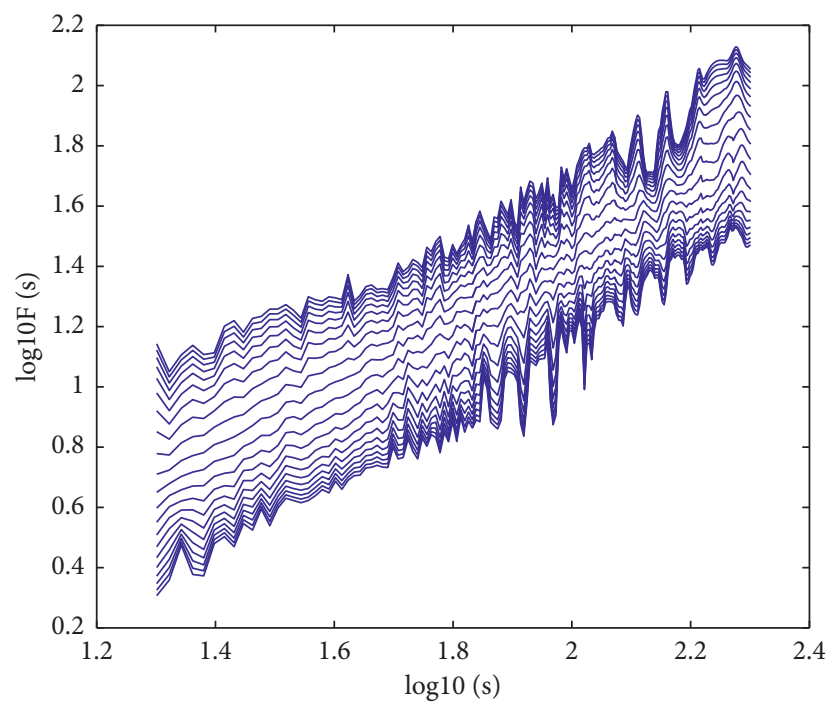

FIGURE 4: $\log -\log$ plot of $F(s)$ versus s for GDCP and USEPU.

starts to climb and reach its peak at $q=4$, showing an S-shaped curve. Also, when $q$ is below zero, the Hurst exponent of GDCP is greater than that of GDCP-USEPU and USEPU, demonstrating the highest level of persistence. On the contrary, when $q$ is above zero, the Hurst exponent of USEPU moves upward sharply and tops the three series correlations. Also, we can find from Figure 5 that none of the curves are flat, indicating the multifractality existence between series.

In addition, we calculate the Rényi exponents, another indicator of multifractality, with the cross-correlation of GDCP-USEPU, the correlation within GDCP series, and the correlation within USEPU series. As presented in Figure 6, we can find that none of three curves present typical linear shapes, proving the existence of the multifractality character between GDCP and USEPU.

Finally, for the measurement of the multifractal richness of the cross-correlations between GDPC and USEPU, we perform another singularity spectrum check as shown in Figure 7. We can find that the none of the singularity spectrums, $f_{X Y}(\alpha)$, demonstrate linear shapes, showing the multifractality between GDPC and USEPU. Moreover, we calculate the difference between min and max Hölder exponent, the delta, as the measurement for the multifractality degree, as presented in Table 2. It is easy to find that the GDCP delta of Hölder exponent is of the largest value (0.930), showing the strongest multifractality character. 


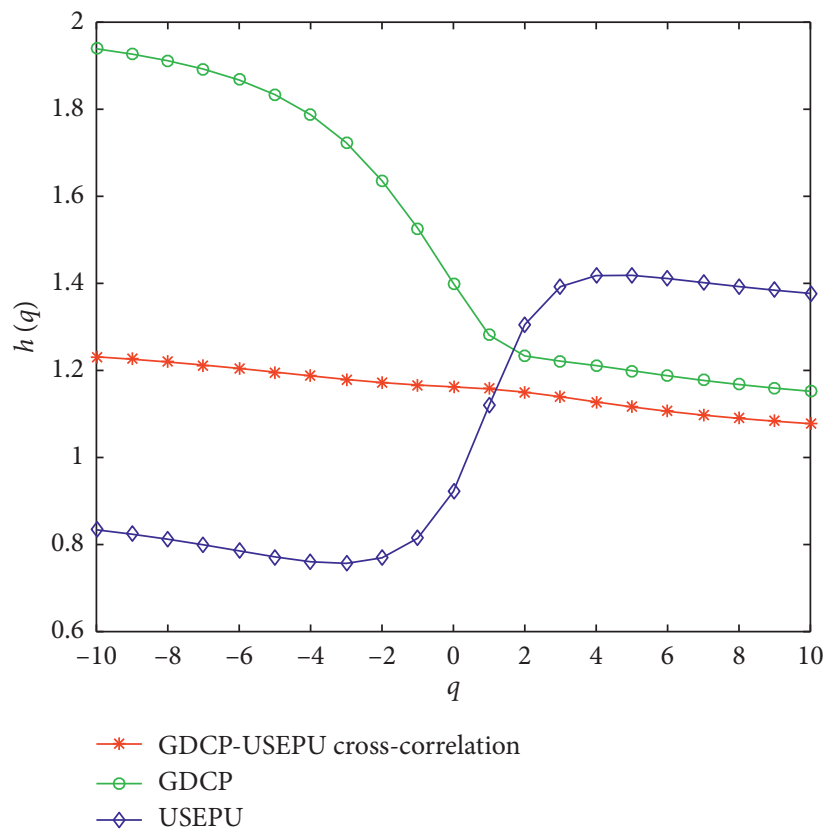

FIGURE 5: Generalized Hurst exponent of $h(q)$ versus q for GDCP and USEPU cross-correlation, the correlation of GDCP, and the correlation of GDCP.

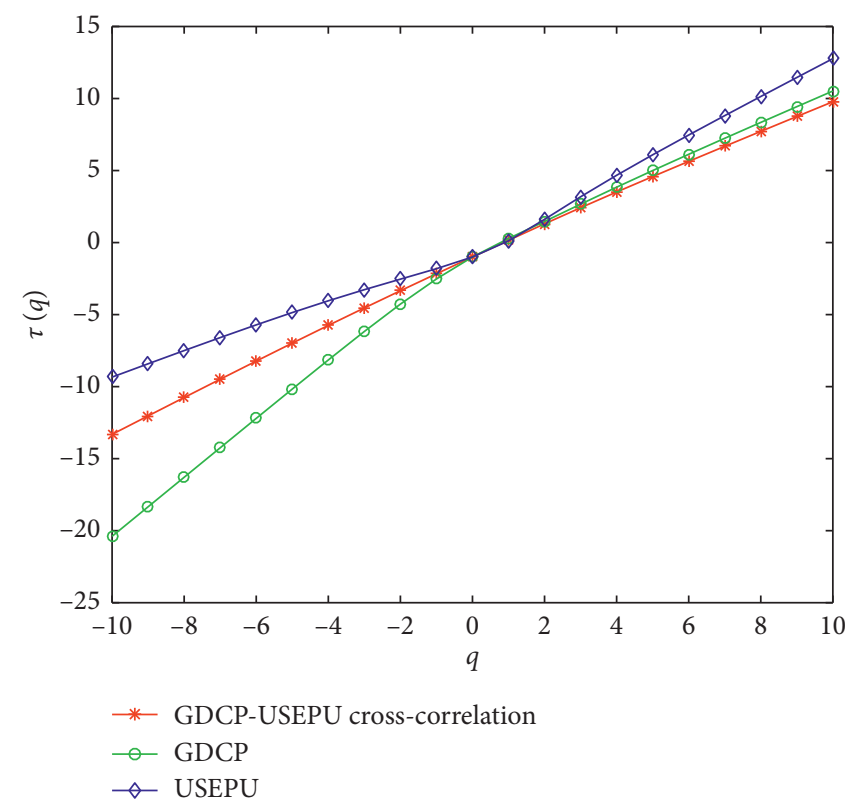

FIGURE 6: Rényi exponent of $\tau$ (q) versus q for GDCP and USEPU cross-correlation, the correlation of GDCP, and the correlation of USEPU. 


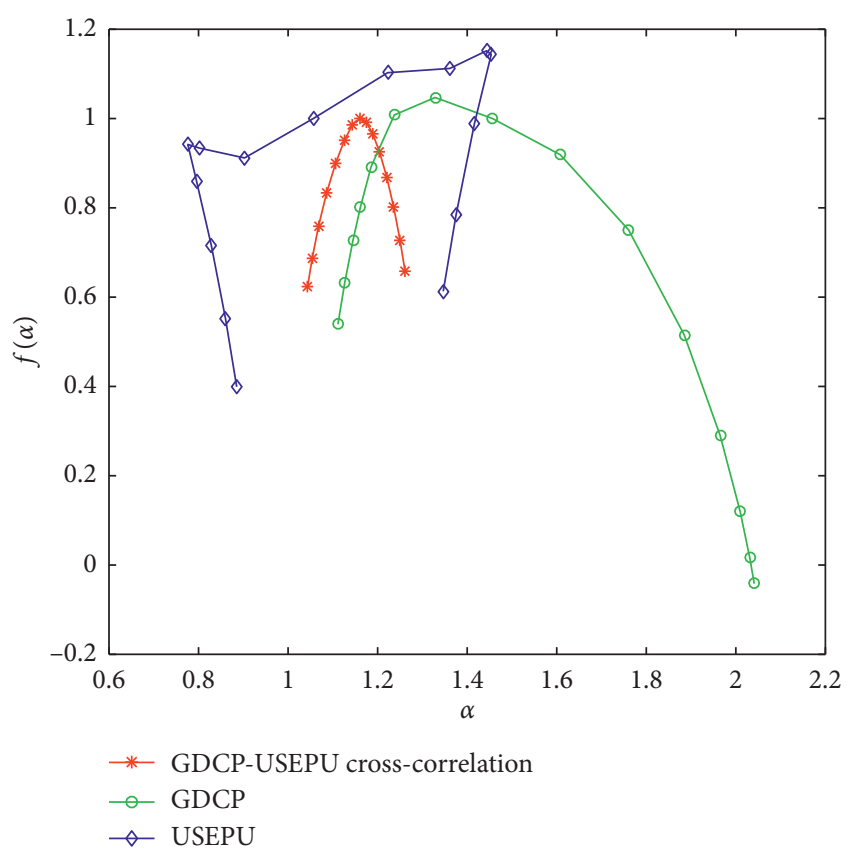

FIGURE 7: Spectrum singularity of $f(\alpha)$ versus $\alpha$ for GDCP and USEPU cross-correlation, the correlation of GDCP, and the correlation of USEPU.

TABle 2: Max, min, and delta values of the Hölder exponent.

\begin{tabular}{lccc}
\hline & $\alpha_{\max }$ & $\alpha_{\min }$ & $\Delta \alpha=\alpha_{\max }-\alpha_{\min }$ \\
\hline GDCP & 2.041 & 1.111 & 0.930 \\
USEPU & 1.454 & 0.776 & 0.678 \\
GDCP-USEPU cross-correlation & 1.261 & 1.044 & 0.218 \\
\hline
\end{tabular}

\section{Conclusions}

In this paper, we take an investigation of the correlation between China's most prosperous carbon emission trading market and US economic policy uncertainty. To have a deep insight of the long-term cross-correlation property between series, we employ the prevailing multifractal detrended cross-correlation analysis as the instrument. We first calculate the cross-correlation statistics $Q_{c c}$ and find sufficient evidence for the correlation significance between Guangdong carbon emission trading price (GDCP) and US economic policy uncertainty (USEPU). Also, to have a more detailed understanding of the nonlinear cross-correlation property between GDCP and USEPU, we calculate the fluctuation function and find that all the function values demonstrate linear shapes, proving the long-term power law cross-correlation between GDCP and USEPU. In addition, we employ the Hurst exponent as the key parameter to check the persistence of correlation between GDCP and USEPU. We find that the parameters are all over the critical values, confirming the correlation persistence between GDCP and USEPU. Moreover, we calculate the Rényi exponent, the core factor in measuring the multifractality, to investigate the multifractal property between GDCP and USEPU. We find that none of the Rényi exponents demonstrate typical linear curves, supporting the multifractality character between GDCP and USEPU. We further perform another spectrum singularity check as enhancement for the multifractality character between GDCP and USEPU. We find that the delta values of the Hölder exponents are all far from zeros, confirming the existence of multifractality between GDCP and USEPU. With this view, multifractality character enables deep insight for the forecast of future movement of the carbon trading price, which would provide substantial suggestions for the carbon trading management of investors.

\section{Data Availability}

We affirm that the carbon emission trading price and economic policy uncertainty data used to support the findings of this study are available at http:// policyuncertainty.com/and Wind Financial Services which is widely subscribed by the financial researchers, respectively.

\section{Conflicts of Interest}

The author declares that there are no conflicts of interest.

\section{Acknowledgments}

This work was supported by the National Natural Science Foundation of China (71901107, 71790594, and U1811462) and Fundamental Research Funds for the Central Universities (2019JDZD16).

\section{References}

[1] Z. Jia and B. Lin, "Rethinking the choice of carbon tax and carbon trading in China," Technological Forecasting and Social Change, vol. 159, Article ID 120187, 2020.

[2] F. Wen, L. Zhao, S. He, and G. Yang, "Asymmetric relationship between carbon emission trading market and stock market: evidences from China," Energy Economics, vol. 91, Article ID 104850, 2020.

[3] Y. Ma, L. Wang, and T. Zhang, "Research on the dynamic linkage among the carbon emission trading, energy and capital markets," Journal of Cleaner Production, vol. 272, Article ID 122717, 2020.

[4] Q. Feng, Z. Wu, and W. Zhang, "Carbon emissions market adjustment in the electricity supply sector: a government perspective," Journal of Cleaner Production, vol. 275, Article ID 123132, 2020.

[5] K. Yan, W. Zhang, and D. Shen, "Stylized facts of the carbon emission market in China," Physica A: Statistical Mechanics and Its Applications, vol. 555, Article ID 124739, 2020.

[6] L. Sun, M. Xiang, and Q. Shen, "A comparative study on the volatility of EU and China's carbon emission permits trading markets," Physica A: Statistical Mechanics and Its Applications, vol. 560, Article ID 125037, 2020.

[7] G. Wang, Q. Zhang, B. Su, B. Shen, Y. Li, and Z. Li, "Coordination of tradable carbon emission permits market and renewable electricity certificates market in China," Energy Economics, vol. 93, Article ID 105038, 2021.

[8] M. Fleschutz, M. Bohlayer, M. Braun, G. Henze, and M. D. Murphy, "The effect of price-based demand response on carbon emissions in European electricity markets: the 
importance of adequate carbon prices," Applied Energy, vol. 295, Article ID 117040, 2021.

[9] T.-T. Feng, R. Li, H.-M. Zhang, X.-L. Gong, and Y.-S. Yang, "Induction mechanism and optimization of tradable green certificates and carbon emission trading acting on electricity market in China, Resources," Conservation and Recycling, vol. 169, Article ID 105487, 2021.

[10] W. Zhang, J. Li, G. Li, and S. Guo, "Emission reduction effect and carbon market efficiency of carbon emissions trading policy in China," Energy, vol. 196, Article ID 117117, 2020.

[11] M.-Z. Deng and W.-X. Zhang, "Recognition and analysis of potential risks in China's carbon emission trading markets," Advances in Climate Change Research, vol. 10, no. 1, pp. 30-46, 2019.

[12] B. Lin and Z. Jia, "What will China's carbon emission trading market affect with only electricity sector involvement? A CGE based study," Energy Economics, vol. 78, pp. 301-311, 2019.

[13] A. Dutta, "Modeling and forecasting the volatility of carbon emission market: the role of outliers, time-varying jumps and oil price risk," Journal of Cleaner Production, vol. 172, pp. 2773-2781, 2018.

[14] X. Fan, X. Lv, J. Yin, L. Tian, and J. Liang, "Multifractality and market efficiency of carbon emission trading market: analysis using the multifractal detrended fluctuation technique," Applied Energy, vol. 251, Article ID 113333, 2019.

[15] Z. Liu, Y. Ye, F. Ma, and J. Liu, "Can economic policy uncertainty help to forecast the volatility: a multifractal perspective," Physica A: Statistical Mechanics and Its Applications, vol. 482, pp. 181-188, 2017.

[16] M. U. Rehman, N. Asghar, and J. Hussain, "Are disaggregate industrial returns sensitive to economic policy uncertainty," Physica A: Statistical Mechanics and Its Applications, vol. 527, Article ID 121301, 2019.

[17] Y. Jiang, Z. Zhu, G. Tian, and H. Nie, "Determinants of within and cross-country economic policy uncertainty spillovers: evidence from US and China," Finance Research Letters, vol. 31, 2019.

[18] C. T. Albulescu, R. Demirer, I. D. Raheem, and A. K. Tiwari, "Does the U.S. economic policy uncertainty connect financial markets? Evidence from oil and commodity currencies," Energy Economics, vol. 83, pp. 375-388, 2019.

[19] N. Antonakakis, D. Gabauer, and R. Gupta, "Greek economic policy uncertainty: does it matter for Europe? Evidence from a dynamic connectedness decomposition approach," Physica A: Statistical Mechanics and Its Applications, vol. 535, Article ID 122280, 2019.

[20] S. R. Baker, N. Bloom, and S. J. Davis, "Measuring economic policy uncertainty*," The Quarterly Journal of Economics, vol. 131, no. 4, pp. 1593-1636, 2016.

[21] X. Wang, Y. Luo, Z. Wang, Y. Xu, and C. Wu, "The impact of economic policy uncertainty on volatility of China's financial stocks: an empirical analysis," Finance Research Letters, vol. 39, Article ID 101650, 2021.

[22] K.-C. Yen and H.-P. Cheng, "Economic policy uncertainty and cryptocurrency volatility," Finance Research Letters, vol. 38, Article ID 101428, 2021.

[23] D. Dang, H. Fang, and M. He, "Economic policy uncertainty, tax quotas and corporate tax burden: evidence from China," China Economic Review, vol. 56, Article ID 101303, 2019.

[24] H.-C. Hsieh, S. Boarelli, and T. H. C. Vu, "The effects of economic policy uncertainty on outward foreign direct investment," International Review of Economics \& Finance, vol. 64, pp. 377-392, 2019.
[25] Y. Jiang, L. He, J. Meng, and H. Nie, "Nonlinear impact of economic policy uncertainty shocks on credit scale: evidence from China," Physica A: Statistical Mechanics and Its Applications, vol. 521, pp. 626-634, 2019.

[26] W. Kang and R. A. Ratti, "Oil shocks, policy uncertainty and stock market return," Journal of International Financial Markets, Institutions and Money, vol. 26, pp. 305-318, 2013.

[27] Z. Li and J. Zhong, "Impact of economic policy uncertainty shocks on China's financial conditions," Finance Research Letters, vol. 35, Article ID 101303, 2020.

[28] Q. T. Tran, "Economic policy uncertainty and cost of debt financing: international evidence," The North American Journal of Economics and Finance, vol. 57, Article ID 101419, 2021.

[29] Y. Wei, "Oil price shocks, economic policy uncertainty and China's trade: a quantitative structural analysis," The North American Journal of Economics and Finance, vol. 48, pp. 20-31, 2019.

[30] P.-F. Dai, X. Xiong, Z. Liu, T. L. D. Huynh, and J. Sun, "Preventing crash in stock market: the role of economic policy uncertainty during COVID-19," Financial Innovation, vol. 7, no. 1, p. 31, 2021.

[31] S. Adams, F. Adedoyin, E. Olaniran, and F. V. Bekun, "Energy consumption, economic policy uncertainty and carbon emissions; causality evidence from resource rich economies," Economic Analysis and Policy, vol. 68, pp. 179-190, 2020.

[32] J. Yu, X. Shi, D. Guo, and L. Yang, "Economic policy uncertainty (EPU) and firm carbon emissions: evidence using a China provincial EPU index," Energy Economics, vol. 94, Article ID 105071, 2021.

[33] S. Zou and T. Zhang, "Multifractal detrended cross-correlation analysis of the relation between price and volume in European carbon futures markets," Physica A: Statistical Mechanics and Its Applications, vol. 537, Article ID 122310, 2020.

[34] F. Ma, Y. Wei, and D. Huang, "Multifractal detrended crosscorrelation analysis between the Chinese stock market and surrounding stock markets," Physica A: Statistical Mechanics and Its Applications, vol. 392, no. 7, pp. 1659-1670, 2013.

[35] M. Pal, P. Madhusudana Rao, and P. Manimaran, "Multifractal detrended cross-correlation analysis on gold, crude oil and foreign exchange rate time series," Physica A: Statistical Mechanics and Its Applications, vol. 416, pp. 452-460, 2014.

[36] M. Dai, J. Hou, J. Gao, W. Su, L. Xi, and D. Ye, "Mixed multifractal analysis of China and US stock index series," Chaos, Solitons \& Fractals, vol. 87, pp. 268-275, 2016.

[37] M. Dai, S. Shao, J. Gao, Y. Sun, and W. Su, "Mixed multifractal analysis of crude oil, gold and exchange rate series," Fractals, vol. 24, Article ID 1650046, 2016.

[38] X. Zhang, L. Yang, and Y. Zhu, "Analysis of multifractal characterization of Bitcoin market based on multifractal detrended fluctuation analysis," Physica A: Statistical Mechanics and Its Applications, vol. 523, pp. 973-983, 2019.

[39] C.-Z. Yao, C. Liu, and W.-J. Ju, "Multifractal analysis of the WTI crude oil market, US stock market and EPU," Physica A: Statistical Mechanics and Its Applications, vol. 550, Article ID 124096, 2020.

[40] M. A. Naeem, S. Farid, R. Ferrer, and S. J. H. Shahzad, "Comparative efficiency of green and conventional bonds preand during COVID-19: an asymmetric multifractal detrended fluctuation analysis," Energy Policy, vol. 153, Article ID 112285, 2021.

[41] X. Ge and A. Lin, "Multiscale multifractal detrended partial cross-correlation analysis of Chinese and American stock 
markets, Chaos," Solitons \& Fractals, vol. 145, Article ID 110731, 2021.

[42] M. M. Ghazani and R. Khosravi, "Multifractal detrended cross-correlation analysis on benchmark cryptocurrencies and crude oil prices," Physica A: Statistical Mechanics and Its Applications, vol. 560, Article ID 125172, 2020.

[43] R. Yan, D. Yue, X. Chen, and X. Wu, "Non-linear characterization and trend identification of liquidity in China's new OTC stock market based on multifractal detrended fluctuation analysis, Chaos," Solitons \& Fractals, vol. 139, Article ID 110063, 2020.

[44] E. Mnif, A. Jarboui, and K. Mouakhar, "How the cryptocurrency market has performed during COVID 19? A multifractal analysis," Finance Research Letters, vol. 36, Article ID 101647, 2020.

[45] L. H. S. Fernandes, F. H. A. de Araújo, and I. E. M. Silva, "The (in) efficiency of NYMEX energy futures: a multifractal analysis," Physica A: Statistical Mechanics and Its Applications, vol. 556, Article ID 124783, 2020.

[46] B. Podobnik and H. E. Stanley, "Detrended cross-correlation analysis: a new method for analyzing two nonstationary time series," Physical Review Letters, vol. 100, Article ID 084102, 2008 .

[47] W.-X. Zhou, "Multifractal detrended cross-correlation analysis for two nonstationary signals," Physical Review E, vol. 77, Article ID 066211, 2008.

[48] W. Zhang, P. Wang, X. Li, and D. Shen, "Multifractal detrended cross-correlation analysis of the return-volume relationship of bitcoin market," Complexity, vol. 2018, Article ID 8691420, 20 pages, 2018. 